\title{
Papillary thyroid carcinoma: case presentation and literature report
}

\begin{abstract}
We present a case of classic papillary thyroid carcinoma in a 35 -year-old patient who initially consulted dermatology for hair loss and dry skin, fatigue, and mild fatigue. The thyroid hormone report was standard, and the neck ultrasound reported two thyroid nodules and cervical lymphadenopathy TIRADS 4 . The BAAF Bethesda V report. The patient underwent total thyroidectomy and left lymphadenectomy I-VI, classifying as pT2 (m) $\mathrm{pN} 1 \mathrm{~b} \mathrm{cM} 0$, followed by adjuvant management with I-131 with the complete biochemical response at six months. In recent decades, thyroid carcinoma has increased in incidence.
\end{abstract}

Keywords: thyroid carcinoma, papillary carcinoma, I-131, case presentation, thyroidectomy, thyroid
Volume 4 Issue 5 - 2020

\author{
Chávez Hernández María Margarita,' \\ Hernández Hoil Lorena Berenice, ${ }^{2}$ Luis \\ Sandoval Jurado, ${ }^{3}$ María Valeria Jiménez $B a ́ e z^{3}$ \\ 'Endocrinologist, Medical Assistant Coordinator of Health \\ Education, JPM Mexican Social Security Institute Cancun, \\ Quintana Roo, Mexico \\ ${ }^{2}$ First Year Resident of Internal Medicine, Regional General \\ Hospital No. 17 Mexican Institute of Social Security. Cancun, \\ Mexico \\ ${ }^{3}$ Head of Medical Benefits Services, Mexican Institute of Social \\ Security Quintana Roo, GRICIQ (Quintana Roo Clinical \\ Research Group), Mexico
}

\begin{abstract}
Correspondence: María Valeria Jiménez Báez, Head of Medical Benefits Services, Mexican Institute of Social Security Quintana Roo. GRICIQ (Quintana Roo Clinical Research Group), Av. Politécnico Nacional s/n entre Tepic y Kinic Cancún, Quintana Roo, Mexico, Tel 9988742354, Email Valeria.jimenezb@imss.gob.mxValeria.jimenezb@gmail. com
\end{abstract}

Received: October 09, 2020 | Published: October 23, 2020

\section{Introduction}

Thyroid carcinoma is the most common neoplasm and is classified according to its histological characteristics into papillary cancer (PTC), follicular cancer (FTC), medullary cancer (MTC), undifferentiated and anaplastic cancer (ATC). ${ }^{1}$ In the United States, it ranks as the twelfth most common, with papillary being the most frequent variant with a 5 -year survival of $98.3 \%$.2 It is more prevalent in women and those with a genetic family history of thyroid disease, while by age, the most affected group is 45-65 years (43.5\%). ${ }^{2}$ In Mexico, cancer is the third cause of mortality ${ }^{3}$ that has been increasing in recent decades. According to the International Agency for Research on Cancer, thyroid carcinoma is in the $3^{\text {rd }}$ place of incidence in 2018 in Mexico with a mortality of $1.1 \% .{ }^{4}$ In Quintana Roo, in a study carried out on this cancer's epidemiological characteristics, the mean age of 44 years was reported, $93 \%$ women, $77.2 \%$ papillary type, $18 \%$ follicular, and $2.2 \%$ undifferentiated and mixed, ${ }^{5}$ which is consistent with world statistics of the disease. The increase in this cancer incidence has been global, ${ }^{6}$ especially in the United States ${ }^{7}$ and South Korea. ${ }^{8}$ Different authors have studied this phenomenon, and it is thought that it is due to the technological advancement of diagnostic methods and the screening carried out in some populations, which is why the latest international guidelines do not recommend routine screening for this neoplasia. ${ }^{9,10}$ Risk factors include a history of head and neck radiation, age younger than 20 years or older than 45 , thyroid nodule greater than $4 \mathrm{~cm}$, new or enlarged cervical tumor, family history of thyroid cancer. ${ }^{1}$ The clinical case presented describes the diagnostic and treatment process of a papillary thyroid carcinoma in which the reason for consultation differs from the usual presentation.

\section{Description of the clinical case}

The case of a 35-year-old patient is presented, a brother with nonspecific thyroid disease refers. No significant pathological history. She had three pregnancies, which required insemination a year before, of which two were lost. She is guided by dermatology due to loss of eyelashes and eyebrows, dry skin and hair, bradypsychia. The patient reports fatigue and fatigue of 1 year of evolution. On physical examination: Weight $54.4 \mathrm{~kg}$, height $1.61 \mathrm{~m}$. , BMI 21; HR 69bpm, RR 18 rpm, RT 99/57, enlargement of the thyroid gland without palpation of the enlarged neck. The laboratory report with normal thyroid and gynecological profiles, thyroglobulin (Tg) $97.3 \mathrm{ng} / \mathrm{mL}$ (3.5-77). The neck ultrasound reported a $32 \times 11 \times 12 \mathrm{~mm}$ right lobe thyroid gland, $37 \times 9 \times 12 \mathrm{~mm}$ left lobe with two hypoechoic nodules both $<1 \mathrm{~cm}$, one of them poorly defined and highly vascularized. Reactive-looking left adenomegaly. Hypoechoic nodule in the left hemicneck in level III, probable metastatic node. TIRADS 4. BAAF was performed with a report of BETHESDA V compatible with suspicion of malignancy, while the lymph node puncture showed no neoplastic cells. Total thyroidectomy was decided with lymphadenectomy I to VI with the histopathological report of multifocal classic papillary thyroid carcinoma (4 foci): $2.2 \times 1.4 \times 0.8 \mathrm{~cm}, 1.4 \times 0.5 \times 0.3 \mathrm{~cm}, 0.8 \times 0.4 \times 0.3 \mathrm{~cm}$ and one smaller than $0.3 \mathrm{~cm}$. Negative margins without vascular invasion. Microscopic extrathyroid extension. Lymphatic invasion present: Level VI 4/9 positive lymph nodes for metastasis, 1 of them with extracapsular extension. Level I to V $2 / 2$ positive lymph nodes. Classified as pT2 (m) pN1b cM0 with an intermediate risk of recurrence. Two months after thyroidectomy, the scintigraphy was positive for residual functional thyroid tissue, administering doses of $150 \mathrm{mCu}$ 
of I-131, continuing with suppressive treatment with levothyroxine. Control studies after six months reported abolished TSH $(0.06 \mu \mathrm{IU}$ / $\mathrm{mL}$ ), Thyroglobulin at $0.1 \mathrm{ng} / \mathrm{mL}$; serum calcium within normal limits. Ultrasound reported an increase in the size of 2 submandibular ganglia without morphological changes. After 6 months of the surgical process, the patient said energy recovery, denying dysphonia and dysphagia.

\section{Discussion}

This patient comes to the outpatient clinic with clinical data suggestive of hypothyroidism who also has a history of infertility and glandular growth. However, the thyroid profile was found with normal levels and a slight elevation of Thyroglobulin, which may be associated with thyroid disease ${ }^{11}$ and is a marker of tumor growth Few cases of thyroid carcinoma present initially due to suspected hypothyroidism without the presence of palpable nodules. Among the patient's antecedents related to thyroid disease, he refers to a brother with unspecified thyroid disease; underwent hormonal treatment and in vitro insemination for infertility the previous year. Multiple studies have associated infertility, and thyroid carcinoma with contradictory results; $;^{12,13,14}$ however, a meta-analysis ${ }^{15}$ refers to an increased risk of thyroid cancer in infertile women undergoing hormonal treatments, regardless of the number of pregnancies.

Thyroid ultrasound is indicated in the study of the thyroid nodule and goiter. ${ }^{11}$ It allows evaluating the extension of the lesions and the cervical nodes since it has been found that it can identify up to 20 to $30 \%$ of abnormal nodes; ${ }^{16}$ however, they can also fail up to $50 \%$ due to thyroid alteration. ${ }^{17}$ In the case of the patient, two nodules $<1 \mathrm{~cm}$, and changes in the left cervical nodes were detected. The thyroid nodule is a pathology whose incidence has increased, among other causes, due to the increase in the use of ultrasonograms, currently using the TIRADS (Thyroid Imaging Reporting and Data System) classification, with $90 \%$ of these being benign. ${ }^{18}$ According to a meta-analysis that included the study of 10,437 thyroid nodules, the ultrasound presents a sensitivity of $79 \%$, and a specificity of $71 \% .{ }^{19}$ Normally, only nodules larger than $1 \mathrm{~cm}$ are evaluated. However, the study is included in those smaller than $1 \mathrm{~cm}$ that present clinical symptoms or lymph node alterations in the imaging study. ${ }^{11}$

The TIRADS classification corresponded to a lesion with moderate suspicion of malignancy corresponding to a risk of around $10-20 \%$. Due to the fact that the nodule did not present microcalcifications, without extrathyroid extension or greater height than width, a fine needle biopsy (FABB) was performed due to the presence of alteration of cervical nodes compatible with malignancy despite being less than one $\mathrm{cm} .{ }^{11}$ Cytological results detected by BAAF are reported according to the Bethesda system established in 2007 to avoid variability in the reports. ${ }^{11}$ The Bethesda system is made up of 6 categories and offers an estimate of the risk of cancer in them. Category V corresponds to an estimated risk of $60-75 \%$ of malignancy, which is indicative of surgical treatment.

The location of the node can be useful for decision-making since malignant nodes are more common in levels III, IV, and VI. ${ }^{11}$ Confirmation of malignant nodes is done in the same way by means of needle-guided biopsy and by thyroglobulin medium in needle wash where a $\mathrm{Tg}$ less than one $\mathrm{ng} / \mathrm{ml}$ decreases the probability of malignancy. The latter works in cases where the imaging study and the BAAF differ. The main objective of a thyroid nodule classified as suspected malignancy is to establish a histological diagnosis, definitive removal of the pathology, and to reduce risks.

The standard treatment is total thyroidectomy and only in some instances of papillary microcarcinoma (less than $1 \mathrm{~cm}$ ) is close monitoring of the case considered a second option. ${ }^{20}$ Lymphadenectomy is performed therapeutically or prophylactically. Therapy is when the nodal disease is identified or suspected by clinical examination or ultrasound. Prophylaxis is controversial. ${ }^{20}$ It is not necessary for most follicular, non-invasive papillary carcinomas and small ones; however, for patients with advanced stages (greater than $4 \mathrm{~cm}$ with or without extrathyroid invasion), the involvement of lateral lymph nodes or if the information will contribute to the planning of future therapy is being considered. Among the complications of the surgery, the most common is hypoparathyroidism, since it occurs transiently in $20 \%$ and permanently in 0.8 to $3 \%$ after total thyroidectomy. ${ }^{21}$ Clinically, there is tetany, perioral paresthesia, and muscle cramps reaching laryngospasm, seizures generalized, and Chvostek and Trousseau signs. Treatment guidelines suggest starting it when the clinical picture is compatible and a serum calcium report lower than $7.8 \mathrm{mg} / \mathrm{dL}$. However, treatment started the day after surgery is accepted as prevention in cases of a high risk of presenting postoperative hypocalcemia in case of complicated surgery, or if the surgeon considers that the parathyroids were compromised. ${ }^{21}$

Once the anatomical piece has been obtained, the histopathological analysis is performed. The report is made according to the AJCC classification in its $8^{\text {th }}$. Edition with which the pathological TNM staging and the prognostic stage of mortality can be performed. ${ }^{22}$ The T corresponds to the primary tumor characteristics where four tumors or foci are found in the case, the largest being greater than $2 \mathrm{~cm}$ but smaller of $4 \mathrm{~cm}$ being a pT2 (m). When cervical lymph node involvement is found, the subclassification depends on the level where they are located, being a pN1b due to having positive lymph nodes outside of story VI or VII. Finally, due to pathology, data of distant metastasis are not confirmed nor clinically, which corresponds to a cM0. For all the above, the patient reaches stage I of AJCC, which is due to the fact that she is at an age under 55 years, and no distant metastases are detected. The American Thyroid Association classification is used to define its risk of recurrence. ${ }^{11}$ The case corresponds to intermediate-risk since it presents microscopic extrathyroid invasion and more than five positive lymph nodes smaller than $3 \mathrm{~cm}$. All of the above is done to define the need for postsurgical treatment with I-131. From intermediate risk, adjuvant therapy with $131 \mathrm{I}$ is indicated as it improves survival in aggressive variants of papillary carcinoma, in those with a tumor greater than $4 \mathrm{~cm}$ with positive lymph nodes and when there is microscopic extrathyroid extension. ${ }^{11}$

For the use of I-131, levothyroxine suspension is required for TSH suppression for four weeks or the use of rhTSH with a TSH goal greater than $30 \mu \mathrm{IU} / \mathrm{ml}$ at the time of the procedure. After this, scintigraphy is performed, which can help detect residual disease; however, it has a low sensitivity for long-term follow-up $(27-55 \%) .{ }^{20}$ Follow-up is done with dynamic staging that focuses on $\mathrm{Tg}$ and TSH measurements every 3 to 6 months in the 1st year and USG of the neck from 6 to 12 months. At each visit, the patient is classified into excellent response, incomplete biochemical response, vague structural response, and indeterminate response. The excellent response occurs when you have thyroglobulin levels less than $0.2 \mathrm{ng} / \mathrm{ml}$ and no suspicious data on ultrasound. In this case, the patient's first follow-up evaluation can be classified as an incomplete biochemical response since she presented a thyroglobulin level greater than $0.2 \mathrm{ng} / \mathrm{ml}$. However, in later assessment, she is in a therapeutic goal. The ultrasound performed two months after surgery allowed determining her dynamic staging and administering the ablative dose of I 131.20. According to her latest follow-up record, the patient has a complete biochemical response, pending an imaging study to perform the full dynamic staging. 


\section{Conclusion}

Thyroid carcinoma is a disease of current relevance since its incidence has been increasing in recent years, it should be suspected not only in patients who present with an asymptomatic thyroid nodule but also in cases of women with a history of the use of hormonal treatments for fertility, where proper management and follow-up allows reducing the recurrence rates of the disease.

\section{Acknowledgments}

None.

\section{Ethical considerations}

During the entire process, it was carried out following the pertinent ethical considerations and with informed consent signed by the patient to perform the various procedures during her treatment.

\section{References}

1. Jameson J, Fauci A, Kasper D. Harrison Principios de Medicina Interna 20va ed. Mc Graw Hill 2019 Mexico. 2019.

2. Howlader N, Noone AM, Krapcho M, et al. SEER Cancer Statistics Review 1975-2017. National Cancer Institute. Bethesda, MD. April 2020.

3. Aldaco F, Pérez P, Cervantes G, et al. Mortalidad por cáncer en México: actualización 2015. Gac Mex Oncol. 2018;17:28-34.

4. International Agency for Research on Cancer. The Global Cancer Observatory: Mexico sheet facts. 2019.

5. Vidaurri Ojeda A, Gómez Hernández J, Chávez Hernández M, et al. Cáncer de tiroides: Caracterización clínica y concordancia de pruebas diagnósticas. Salud Quintana Roo. 2016;9(33):11-17.

6. Davies L, Morris LG, Haymart M, et al. American Association of Clinical Endocrinologists and American College of Endocrinology disease state clinical review: the increasing incidence of thyroid cancer. Endocr Pract. 2015;21:686-696

7. Powers AE, Marcadis AR, Lee M, et al. Changes in Trends in Thyroid Cancer Incidence in the United States, 1992 to 2016. JAMA. 2019;322(24):2440-2441.

8. Ahn HS, Welch HG. South Korea's thyroid-cancer "epidemic" turning the tide. N Engl J Med. 2015;373(24):2389-2390.

9. Vaccarella S, Dal Maso L, Laversanne M, Bray F, et al. The impact of diagnostic changes on the rise in thyroid cancer incidence: a population based study in selected high-resource countries. Thyroid. 2015; 25: $1127-1136$.
10. Vaccarella S, Franceschi S, Bray F, Wild CP, et al. Worldwide ThyroidCancer Epidemic? The Increasing Impact of Overdiagnosis. $N$ Engl J Med. 2016;375(7):614-617.

11. Haugen BR, Alexander EK, Bible KC, et al. 2015 American Thyroid Association Management Guidelines for Adult Patients with Thyroid Nodules and Differentiated Thyroid Cancer: The American Thyroid Association Guidelines Task Force on Thyroid Nodules and Differentiated Thyroid Cancer. Thyroid. 2016;26(1):1-133.

12. Reigstad MM, Storeng R, Myklebust TÅ, et al. Cancer Risk in Women Treated with Fertility Drugs According to Parity Status-A Registry-based Cohort Study. Cancer Epidemiol Biomarkers Prev. 2017;26(6):953-962.

13. Brinton LA, Moghissi KS, Scoccia B, et al. Effects of fertility drugs on cancers other than breast and gynecologic malignancies. Fertil Steril. 2015;104(4):980-988.

14. Momenimovahed Z, Taheri S, Tiznobaik A, et al. Do the Fertility Drugs Increase the Risk of Cancer? A Review Study. Front Endocrinol (Lausanne). 2019;10:1-13.

15. Yu Q, Lv X, Liu K, et al. Fertility Drugs Associated with Thyroid Cancer Risk: A Systematic Review and Meta-Analysis. Biomed Res Int. 2018; 2018:1-7.

16. Li L, Cheng SN, Zhao YF, et al. Diagnostic accuracy of single-source dual-energy computed tomography and ultrasonography for detection of lateral cervical lymph node metastases of papillary thyroid carcinoma. $J$ Thorac Dis. 2019;11(12):5032-5041.

17. Napolitano G, Romeo A, Vallone G, et al. How the preoperative ultrasound examination and BFI of the cervical lymph nodes modify the therapeutic treatment in patients with papillary thyroid cancer. $B M C$ Surg. 2013;13(2):1-7.

18. Cabanillas ME, McFadden DG, Durante C. Thyroid cancer. Lancet. 2016;388(10061):2783-2795.

19. Wei X, Li Y, Zhang S, et al. Meta-analysis of thyroid imaging reporting and data system in the ultrasonographic diagnosis of 10,437 thyroid nodules. Head Neck. 2016;38(2):309-315.

20. Filetti S, Durante C, Harti D, et al. Thyroid cancer: ESMO clinical practice guidelines for diagnosis, treatment, and follow up. Ann Oncol. 2019;30(12):1856-1883.

21. Orloff LA, Wiseman SM, Bernet VJ, et al. American Thyroid Association Statement on Postoperative Hypoparathyroidism: Diagnosis, Prevention, and Management in Adults. Thyroid. 2018;28(7):830-841.

22. American Joint Commission on Cancer. AJCC Cancer Staging Manual. $8^{\text {th }}$ ed. Springer International Publishing, 2017. EUA. 